\title{
Highly thermally conductive carbon nanotubes pillared exfoliated graphite/polyimide composites
}

\author{
Yongqiang Guo ${ }^{1}$, Shuangshuang Wang ${ }^{1}$, Kunpeng Ruan ${ }^{1}$, Haitian Zhang ${ }^{1}$ and Junwei Gu iD $^{\text {凶 }}$
}

In this work, carbon nanotubes pillared grew on exfoliated graphite by the microwave-assisted method is utilized as thermally conductive fillers (CPEG) in polyimide (PI) to fabricate CPEG/PI thermally conductive composites with the combining ways of "in-situ polymerization, electrospinning, lay-up, and hot-pressing". The prepared CPEG/PI composites realized the maximum thermal conductivity $\left(\lambda, 1.92 \mathrm{~W} \mathrm{~m}^{-1} \mathrm{~K}^{-1}\right)$ at low CPEG amount (10 wt\%), much higher than that of pure PI $\left(0.28 \mathrm{~W} \mathrm{~m}^{-1} \mathrm{~K}^{-1}\right)$. The $\lambda$ of CPEG/PI composites show almost no change after 1000 cycles of heating and cooling at the temperature of $25-100^{\circ} \mathrm{C}$. The finite element analysis simulates the nano-/microscale heat transfer in CPEG/PI composites to reveal the internal reason of the $\lambda$ enhancement. The improved thermal conductivity model and empirical equation could better reflect the actual $\lambda$ change trend of CPEG/PI composites. The actual application test shows the CPEG/PI composites could significantly reduce the operating temperature of the CPU in mobile phone.

npj Flexible Electronics (2021)5:16; https://doi.org/10.1038/s41528-021-00113-z

\section{INTRODUCTION}

The miniaturization, lightness, and intelligence of consumer electronics, the large-scale construction of communication base station brought about by $5 \mathrm{G}$ commercialization, and the rapid development of batteries in new energy vehicle (NEV) and other fields have all put forward higher requirements for highly thermally conductive materials ${ }^{1-3}$. Polymer-based composites are used ubiquitously in the above areas ranging from substrate, frame to interlayer medium, and sealing material, due to their lightweight, easy processing, and excellent chemical stability ${ }^{4,5}$. However, the intrinsic low thermal conductivity $(\lambda)$ of polymers cannot meet the increasing heat conduction and dissipation demand in the fields of high-end electronic, 5G, and NEV, etc. ${ }^{6,7}$.

At present, there are two main ways to improve the $\lambda$ of polymer materials. One is synthesizing new-type polymers with high intrinsic $\lambda$ (such as liquid crystal epoxy and polyimide ${ }^{8-10}$, triphenylene-based discotic liquid crystal polymers ${ }^{11}$, conjugated poly(3-hexylthiophene) ${ }^{12}$, etc.), the other is adding highly thermally conductive fillers (such as graphene ${ }^{13}$, carbon nanotubes $(\mathrm{CNT})^{14}$, boron nitride nanosheets (BNNS) ${ }^{15}$, etc.) into the polymer matrix. The latter is simple, efficient, and suitable for industrial production ${ }^{16,17}$. However, while ensuring the required thermal conductivity, the amount of thermally conductive fillers should be reduced as much as possible to maintain the inherent advantages of polymer-based composites such as lightweight, easy processing, and excellent mechanical properties ${ }^{5}$.

One-dimensional (1D) CNT and two-dimensional (2D) graphite derivatives are favored by researchers in the field of thermally conductive fillers due to their ultra-high $\lambda^{18-20}$. By now, a number of researchers directly adding CNT and graphite derivatives into the polymer to improve the $\lambda$ of the polymer-based composites ${ }^{21,22}$. Wang et al. ${ }^{23}$ blended graphene nanoplatelets (GNP), CNT and polyvinylidene fluoride (PVDF) to prepare CNT/GNP/PVDF thermally conductive composites. When the amount of CNT/GNP was 22 wt $\%$ (CNT:GNP = 1:10, wt:wt), the $\lambda$ of the CNT/GNP/PVDF thermally conductive composites reached $1.92 \mathrm{~W} \mathrm{~m}^{-1} \mathrm{~K}^{-1}$, while the $\lambda$ of GNP/PVDF and CNT/PVDF thermally conductive composites prepared with the same amount of fillers were 1.64 and $0.56 \mathrm{~W} \mathrm{~m}^{-1} \mathrm{~K}^{-1}$, respectively. Kim et al. ${ }^{24}$ prepared multiwalled CNT/GNP/polycarbonate (MWCNT/GNP/PC) thermally conductive composites by the blending method, and confirmed that the MWCNT/GNP hybrid fillers had better thermal conductivity improvement effect than MWCNT or GNP single fillers. When the amount of fillers was $20 \mathrm{wt} \%$, the $\lambda$ of MWCNT/GNP/PC, GNP/ $\mathrm{PC}$, and MWCNT/PC thermally conductive composites were 1.39, 1.13 , and $0.74 \mathrm{~W} \mathrm{~m}^{-1} \mathrm{~K}^{-1}$, respectively, mainly attributed to the synergistic effect of the line-surface structure of CNT/graphite derivative hybrid fillers ${ }^{25}$. The 1D CNT can be used as the basic unit to bridge adjacent 2D graphite derivative sheets, constructing more effective thermal conduction pathways ${ }^{26}$. The introduction of 2D graphite derivatives can effectively promote the uniform dispersion of CNT in the polymer matrix, benefiting the formation of thermal conduction pathways with relatively low fillers amount ${ }^{27}$. However, it is also found that the simple blending of CNT and graphite derivatives has limited enhancement in the $\lambda$ of polymer-based composites. On the one hand, the bridging effect is limited, and it is easily destroyed during the blending process. On the other hand, the agglomeration problem of graphite derivatives and CNT cannot be solved effectively ${ }^{28}$.

In order to solve the above problems better, researchers have tried to connect CNT and graphite derivatives by chemical or physical interaction. Li et al. ${ }^{29}$ built a kind of thermally conductive fillers with hierarchical structure by electrostatic self-assembly between the positively charged (3-aminopropyl)trimethoxysilane (APTMS) modified CNT (A-CNT) and negatively charged graphene oxide (GO), then fabricated GO-A-CNT/PVDF thermally conductive composites by solution compounding method. When the amount of GO-A-CNT was $10 \mathrm{wt} \%$ and the mass ratio of A-CNT to GO was $4: 1$, the $\lambda$ of GO-A-CNT/PVDF composites reached $1.56 \mathrm{~W} \mathrm{~m}^{-1} \mathrm{~K}^{-1}$, the $\lambda$ enhancement of $610 \%$ was achieved compared with that of pure PVDF. However, the $\lambda$ of $10 \mathrm{wt} \%$ CNT/PVDF composites was only $0.43 \mathrm{~W} \mathrm{~m}^{-1} \mathrm{~K}^{-1}$. Zhao et al. ${ }^{30}$ prepared a kind of hybrid fillers

${ }^{1}$ MOE Key Laboratory of Material Physics and Chemistry under Extraordinary Conditions, Shaanxi Key Laboratory of Macromolecular Science and Technology, School of Chemistry and Chemical Engineering, Northwestern Polytechnical University, Xi'an, Shaanxi, People's Republic of China. ${ }^{凶}$ email: gjw@nwpu.edu.cn 
by utilizing the reaction between dodecylamine-modified graphene nanoplatelets (DA-GNP) and $\gamma$-aminopropyl-triethoxysilane treated MWCNT ( $f$-MWCNT), and then fabricated DA-GNS/fMWCNT/cyanate ester resin (CE) thermally conductive composites. The results showed that chemical modification significantly improved the dispersion performance of $f$-MWCNT/DA-GNP, and led to an effective decrease in the interfacial thermal resistance. When the amount of DA-GNS/f-MWCNT was $5 \mathrm{wt} \%$, the $\lambda$ of (DAGNS/f-MWCNT)/CE composites reached $0.86 \mathrm{~W} \mathrm{~m}^{-1} \mathrm{~K}^{-1}, 3.2$ times higher than the pure CE matrix $\left(0.26 \mathrm{~W} \mathrm{~m}^{-1} \mathrm{~K}^{-1}\right)$. The $\lambda$ of $f$ MWCNT/CE and DA-GNS/CE thermally conductive composites were only 0.65 and $0.40 \mathrm{~W} \mathrm{~m}^{-1} \mathrm{~K}^{-1}$, respectively. In our previous research works, Gu et al. ${ }^{31}$ grafted aminated MWCNT to the surface of GO and reduced it to obtain $f$-MWCNT-g-rGO thermally conductive fillers, and then fabricated $f$-MWCNT- $g$-rGO/polyimide (PI) thermally conductive composites via "in-situ polymerization, electrospinning, lay-up and hot-pressing" technique. When the amount of $f$-MWCNT- $g$-rGO was $10 \mathrm{wt} \%$ and the mass ratio of $f$ MWCNT to rGO was $1: 2$, the $\lambda$ of $f$-MWCNT- $g$-rGO/PI thermally conductive composites reached $1.60 \mathrm{~W} \mathrm{~m}^{-1} \mathrm{~K}^{-1}$, much higher than the $\lambda\left(1.21 \mathrm{~W} \mathrm{~m}^{-1} \mathrm{~K}^{-1}\right)$ of the $\mathrm{rGO} / \mathrm{PI}$ composites at the same amount of rGO.

It can be seen that the method of modifying and chemical bonding CNT and graphite derivatives can effectively solve the agglomeration problems of CNT and graphite derivatives, which is to the benefit of improving the $\lambda$ of polymer-based composites compared with directly mixing thermally conductive fillers and polymer matrix. However, most of these methods require modification of CNT or graphite derivatives which will reduce their intrinsic $\lambda$ to a certain extent ${ }^{31}$. In addition, the CNT mostly stack on the graphite derivative sheets in a disordered manner (the CNT axis is parallel to the graphite derivative plane) in the CNT/graphite derivative hybrid fillers prepared by directly blending or chemical bonding ${ }^{32}$. However, phonons in CNT and graphite derivatives easily transfer in the axial or in-plane direction, and scatter severely in the radial or through-plane direction due to the $\mathrm{sp}^{2}$ hybrid structure of carbon atoms ${ }^{33}$. Therefore, the structure that CNT randomly stack on the graphite derivative sheets makes the CNT/graphite derivative hybrid fillers have low through-plane $\lambda\left(\lambda_{\perp}\right)$.

An effective method to solve the above problems is making CNT pillared grow on the surface of the graphite derivative to construct three-dimensional (3D) structured thermally conductive fillers. The structure of CNT pillared standing on the surface of the graphite derivative can effectively reduce the thermal resistance at the junction between CNT and graphite derivative, avoiding heat scattering caused by the overlap among the CNT, giving full play to the ultra-high axis $\lambda$ of CNT and in-plane $\lambda$ of graphite derivative ${ }^{34}$. All the above advantages will realize a marked increase in the $\lambda$ of polymer-based thermally conductive composites under the condition of low amount of thermally conductive fillers. Liu et al. ${ }^{34}$ used the chemical vapor deposition (CVD) method, realizing CNT vertically grew on the surface of graphene. The calculation results showed the interfacial thermal resistance between CNT and graphene decreased from $7.5 \times 10^{-7}$ $\mathrm{m}^{2} \mathrm{~K}^{-1} \mathrm{~W}^{-1}$ under natural contact conditions to $9 \times 10^{-10} \mathrm{~m}^{2} \mathrm{~K}^{-1}$ $\mathrm{W}^{-1}$. Feng et al. ${ }^{35}$ also utilized the CVD method to make CNT grew on the surface of expanded graphite, and the $\lambda_{\perp}\left(38 \mathrm{~W} \mathrm{~m}^{-1} \mathrm{~K}^{-1}\right)$ was $170 \%$ higher than that of pure expanded graphite. However, most of the 3D-structured CNT/graphite derivatives that have been reported so far were prepared based on the CVD method, which has disadvantages such as low efficiency, high cost, and high demands of reaction conditions ${ }^{36,37}$.

The microwave-assisted method does not require strict experimental conditions, and can greatly shorten the reaction time (only tens of seconds) to prepare 3D-structured CNT/graphite derivatives fillers ${ }^{38}$. At present, a number of carbon nanomaterials have been synthesized by the microwave-assisted technique ${ }^{38,39}$.
However, the CNT in the 3D-structured CNT/graphite derivatives fillers prepared by the microwave-assisted method mostly distributes in the form of random coils, the dispersibility and $\lambda_{\perp}$ are not ideal. It is still a challenging and valuable work to prepare 3D-structured CNT/graphite derivative thermally conductive fillers with CNT vertically grown on the graphite derivative sheets by the microwave-assisted method. In this paper, the 3D-structured thermally conductive fillers with CNT pillared grown on the surface of exfoliated graphite (CPEG) were prepared simply and efficiently by the microwave-assisted method. The effects of microwave reaction time, gas atmosphere, and raw material ratio on the morphological characteristics, orientation degree, crystal structure, etc. of CPEG were studied in detail. Scanning electron microscope (SEM), transmission electron microscope (TEM), Raman spectroscopy (Raman), and X-ray diffraction (XRD) were used to characterize the morphologies and structures of CPEG thermally conductive fillers, and the microwave-assisted method was optimized according to the orientation of CNT in CPEG. The optimized CPEG was used to fabricate CPEG/PI thermally conductive composites by the ways of "in-situ polymerization, electrospinning, lay-up, and hot-pressing". The effect of the amount of CPEG on the $\lambda$, glass transition temperature $\left(T_{\mathrm{g}}\right)$, heat resistance temperature $\left(T_{\mathrm{HRI}}\right)$, and other properties of CPEG/PI thermally composites were studied in detail. Finite element analysis was used to simulate the nano-/microscale heat transfer in CPEG/PI thermally conductive composites, analyzing the essential reasons that CPEG significantly improves the $\lambda$ of CPEG/PI thermally conductive composites from the microscopic perspective. Based on the effective medium theory and the first law of thermodynamics, a more widely applicable thermal conductivity model and equation was established. On this basis, the $\lambda$ stability of the CPEG/PI thermally conductive composites was verified by repeatedly testing the $\lambda$ in the heating-cooling process, and the actual application test of the CPU in mobile phone was used to analyze the heat conduction and dissipation capability of CPEG/PI thermally conductive composites.

\section{RESULTS AND DISCUSSION \\ Preparation of CPEG and CPEG/PI thermally conductive composites}

The preparation schematic diagram of CPEG and CPEG/PI thermally conductive composites are shown in Fig. 1. EG is prepared by liquid-phase exfoliation of pre-expanded graphite, and its lateral size $(\sim 5.5 \mu \mathrm{m})$ and thickness $(\sim 0.2 \mu \mathrm{m})$ are smaller than that of graphite (lateral size of $\sim 8.5 \mu \mathrm{m}$, thickness of $\sim 0.7 \mu \mathrm{m})$. Besides, the surface of EG is smooth and there are no impurities (Supplementary Fig. 2), indicating that the method used in this paper can effectively exfoliate and purify graphite. After microwave treatment, the appearance of EG is more fluffy and the color is darkened (Supplementary Fig. 1a). Both PAA and CPEG/ PAA fibers (Supplementary Fig. 1b, c) prepared by electrospinning have a high degree of orientation. The apparent color of CPEG/PI composites deepens with the increase of the amount of CPEG (Supplementary Fig. 1d).

\section{Characterization of CPEG}

In order to efficiently and stably prepare 3D-structured thermally conductive fillers with CNT pillared grown on the surface of EG (CPEG), this work optimized the gas atmosphere, reaction time, and the mass ratio of $F C$ to $E G$ during the microwave reaction process. In the air atmosphere, almost no CNT grows on the surface of EG (Supplementary Fig. 3). Considering the hydrogen $\left(\mathrm{H}_{2}\right)$ can regulate the growth of CNT, but it is flammable and dangerous, this work uses hydrogen/argon $\left(\mathrm{H}_{2} / \mathrm{Ar}\right)$ mixture gas (volume fraction of $\mathrm{H}_{2}$ is $5 \%$ ). In general, CNT can be grown under the given reaction time and the mass ratio of $\mathrm{FC}$ and $\mathrm{EG}$ 


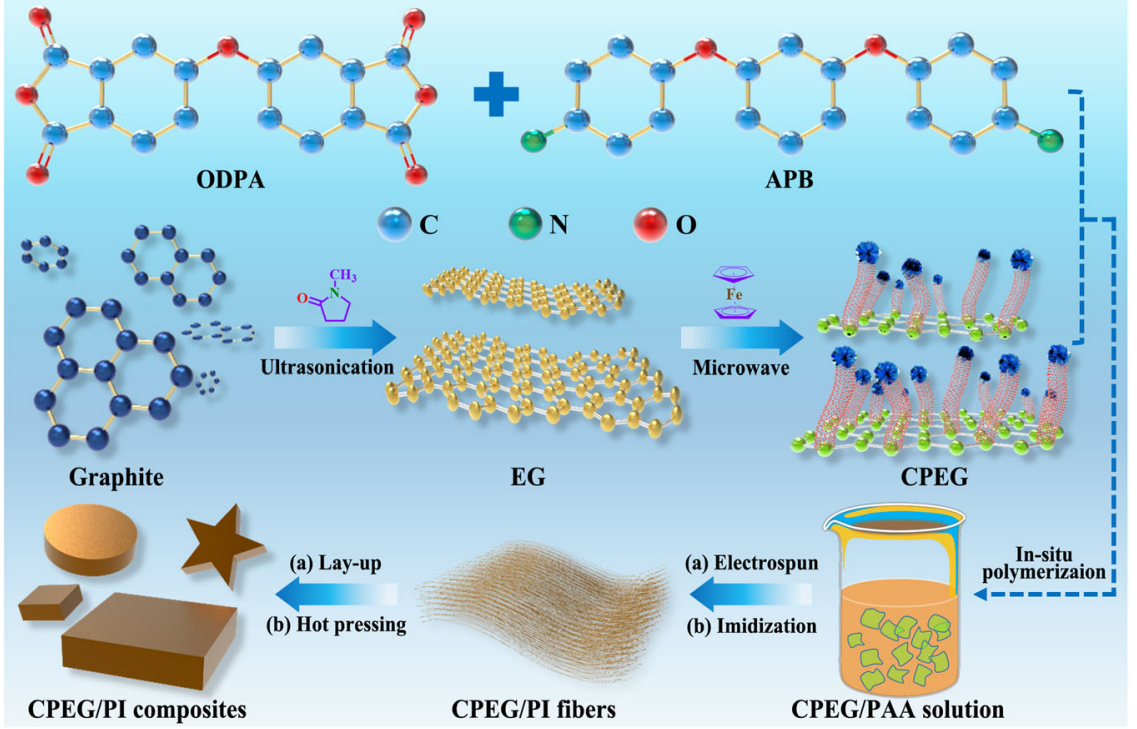

Fig. 1 Scheme of the preparation process. Preparation schematic diagram of CPEG thermally conductive fillers and CPEG/PI thermally conductive composites.
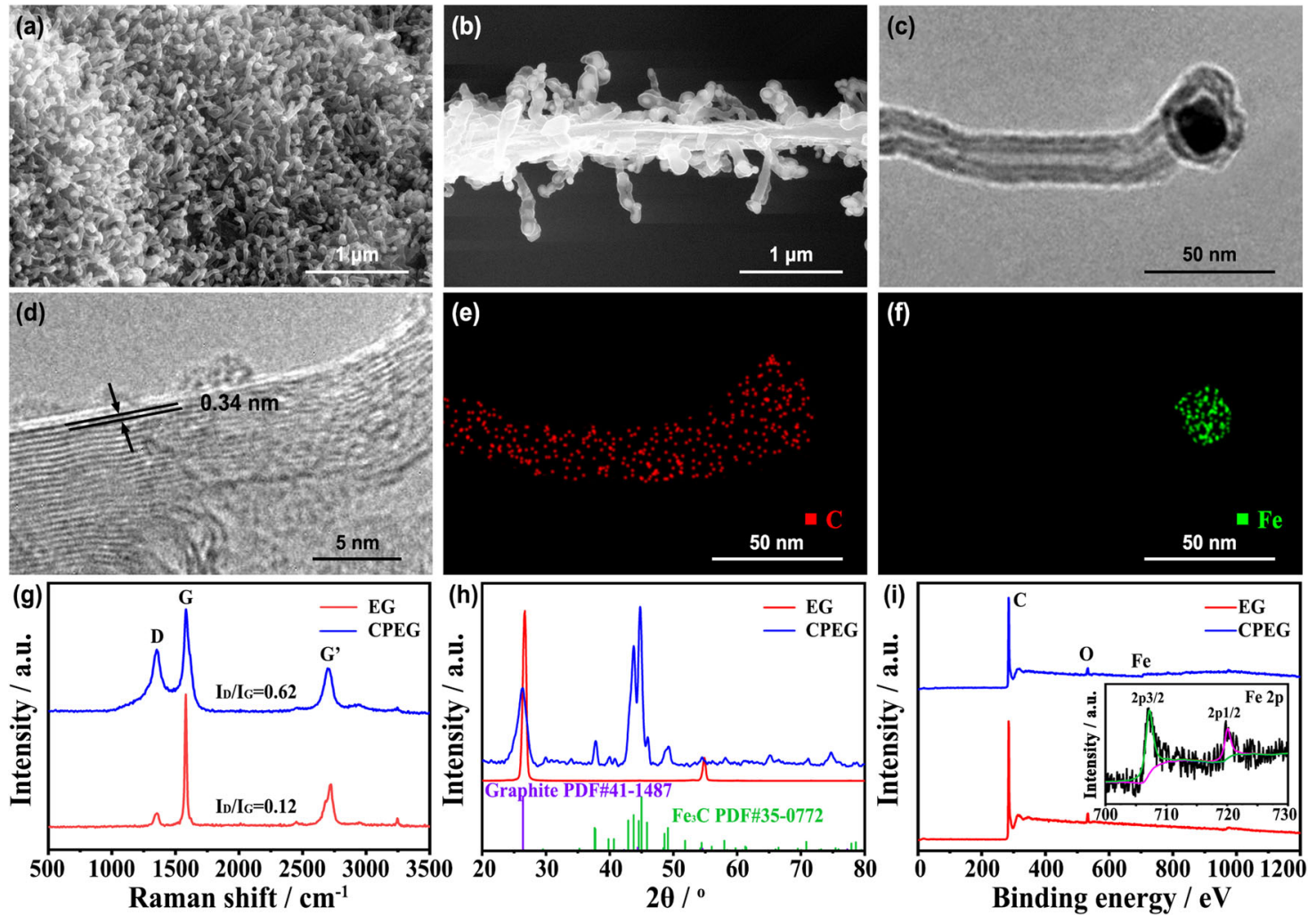

Fig. 2 Characterization of CPEG. SEM images (a, b), TEM images (c, d), EDS mapping images (e, f), Raman spectrum (g), XRD spectrum (h) and XPS spectrum (i).

(Supplementary Fig. 4). The length of CNT increases with the increase of FC proportion and reaction time. When the mass ratio of FC to EG is greater than 0.25 and the reaction time is $90 \mathrm{~s}$, the CNT distributes on the surface of EG like random coils. When the mass ratio of FC to EG is 0.5 and the reaction time is $60 \mathrm{~s}$, CNT all pillared stands on the surface of EG and has obvious alignment structures. The length and diameter of CNT are approximately 600 and $50 \mathrm{~nm}$, respectively. Therefore, the optimal growth conditions for CPEG determined in this paper are $\mathrm{H}_{2} / \mathrm{Ar}$ mixture gas, the reaction time of $60 \mathrm{~s}$, and the mass ratio of FC to EG of 0.5 . The CPEG mentioned later is prepared under this condition.

Figure 2 shows the structure and morphology characterization of CPEG. As shown in the SEM images of Fig. 2a, CNT uniformly pillared stands on the surface of EG, and there is a white particle at its top. The roots of CNT embed on the surface of EG, and both sides of the EG sheet have CNT growth, and some CNT contains particles in the middle part (Fig. 2b). Figure 2c clearly shows the CNT has a multi-walled structure, and the CNT completely coats 

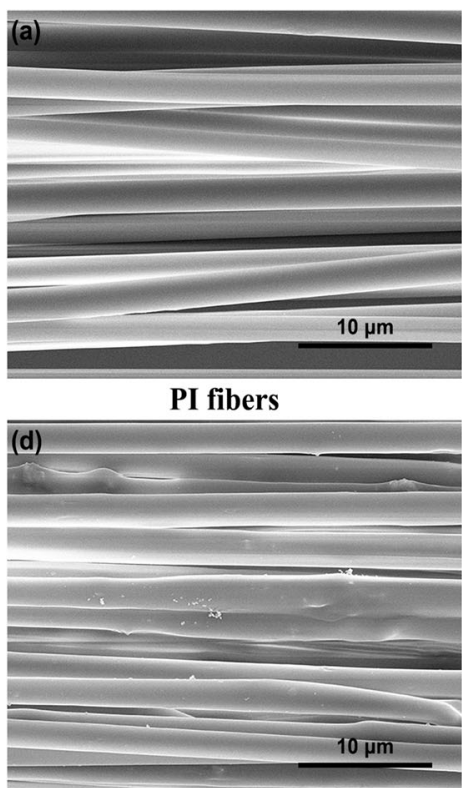

$5 \mathrm{wt} \%$ CPEG/PI fibers

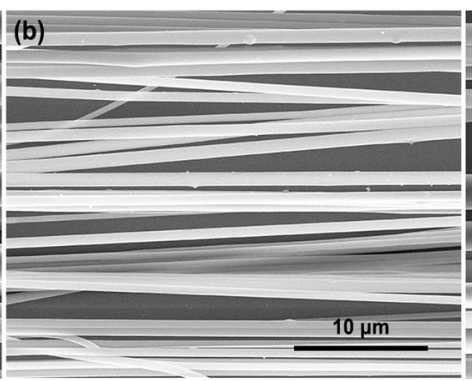

$1 \mathrm{wt} \%$ CPEG/PI fibers

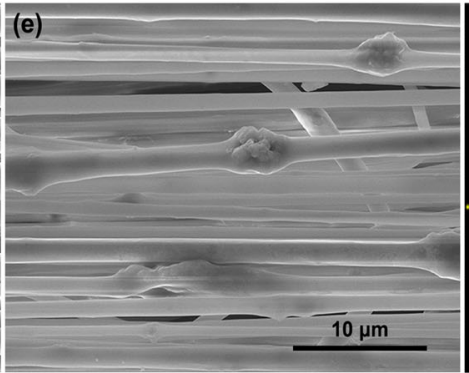

$10 \mathrm{wt} \%$ CPEG/PI fibers

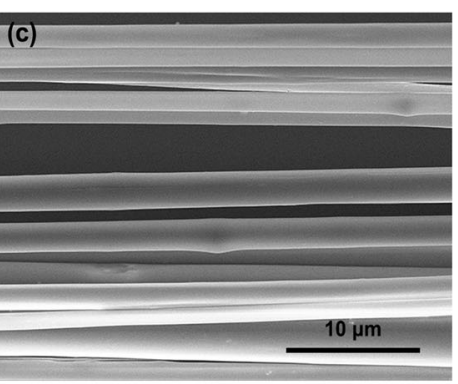

3 wt\% CPEG/PI fibers

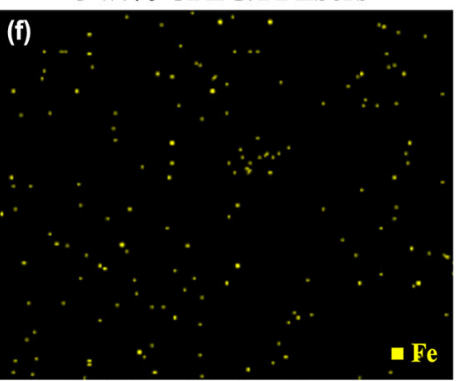

EDS of $10 \mathrm{wt} \%$ CPEG/PI fibers

Fig. 3 SEM images of electrospun fibers. Pure PI fibers (a), CPEG/PI fibers with the amount of CPEG is 1 wt $\%$ (b), 3 wt $\%$ (c), 5 wt $\%$ (d), and 10 wt\% (e), EDS mapping images of 10 wt $\%$ CPEG/PI fibers (f).

the particle. High magnification TEM (Fig. 2d) shows that the wall spacing of CNT is $0.34 \mathrm{~nm}$, which corresponds to the (002) lattice plane of the graphene carbon phase ${ }^{40}$. The EDS elemental mapping images of CPEG (Fig. 2e, $f$ ) shows the homogeneous distribution of $\mathrm{C}$ element throughout the nanotube and the particle contains the iron element. There are three main characteristic peaks in the Raman spectrum of CPEG (Fig. $2 \mathrm{~g}$ ). The $D$ peak $\left(\sim 1350 \mathrm{~cm}^{-1}\right)$ is a disordered vibration peak, indicating defects in the carbon atom lattice. The $G$ peak $\left(\sim 1580 \mathrm{~cm}^{-1}\right)$ is caused by the in-plane vibration of $\mathrm{sp}^{2}$ carbon atoms, reflecting the degree the close-packed hexagonal graphite crystal structure of carbon atoms. The $\mathrm{G}^{\prime}$ peak $\left(\sim 2700 \mathrm{~cm}^{-1}\right)$ is two-phonon resonance second-order Raman peak, which is used to characterize the stacking of carbon atoms in the sample ${ }^{41}$. Generally, the intensity ratio of $D$ peak to $G$ peak $\left(I_{D} / I_{G}\right)$ is used to indicate the degree of disorder. The value $I_{D} / I_{G}(0.62)$ of CPEG is significantly higher than that of EG (0.12), mainly attributed to that the CNT on the surface of EG, disturbs the in-plane vibration of the EG carbon atoms. At the same time, defects are inevitably introduced into CNT during its growth process ${ }^{42}$, resulting in increased defects in the overall structure of CPEG. XRD is used to further characterize the crystal structure of CPEG (Fig. 2h). Both EG and CPEG have obvious diffraction peaks at $2 \theta=26.38^{\circ}$, corresponding to the (002) lattice plane of graphite. It shows that the crystal structure of graphite has no change during the pre-expansion, ultrasonication exfoliating, and microwave treatment process. The remaining diffraction peaks in the XRD spectrum of CPEG are consistent with the standard diffraction peaks of $\mathrm{Fe}_{3} \mathrm{C}$, indicating that the particles in CPEG contain $\mathrm{Fe}_{3} \mathrm{C}$. Therefore, the growth mechanism of CNT in CPEG can be inferred: EG heats up rapidly in the microwave field, and the $\mathrm{H}_{2}$ promotes the decomposition of FC to produce metallic iron and hydrocarbons. After that, the carbon source dissolves into the iron particles to form $\mathrm{Fe}_{3} \mathrm{C}$. After saturation of the carbon in the iron particles, solid carbon precipitates outward and grows into $\mathrm{CNT}^{43}$. Furthermore, XPS (Fig. 2i) is carried out to determine the element composition and the chemical state of CPEG. The signals of $\mathrm{C}, \mathrm{O}$, and Fe are evidenced at $\sim 284, \sim 533$, and $\sim 708 \mathrm{eV}$. Among the signals, the narrow XPS spectrum of Fe $2 p$ exhibits two peaks at 707.5 and $720.2 \mathrm{eV}$ (inset in Fig. 2i) corresponding to the $\mathrm{Fe} 2 \mathrm{p}_{3 / 2}$ and $\mathrm{Fe} 2 \mathrm{p}_{1 / 2}$ spin-orbit peaks of $\mathrm{Fe}$, confirming the formation of $\mathrm{Fe}$ in $\mathrm{CPEG}^{44}$. Besides, EG has a high carbon/oxygen atom concentration ratio $(\sim 32.2)$, indicating that the preexpansion and ultrasonication exfoliating process did not cause excessive oxidation of graphite.

\section{Morphologies of CPEG/PI fibers and composites}

SEM images of CPEG/PI fibers are shown in Fig. 3. The pure PI fibers have a smooth surface and uniform diameter distribution (Figs. 3a, 2.2-2.5 $\mu \mathrm{m}$ ). With the increase of the amount of CPEG, the diameter of CPEG/PI fibers decrease firstly and then increases (Fig. 3b-e). The main reason is that the electrical conductivity and viscosity of the CPEG/PAA solution increase with the amount of CPEG increases. (Due to the CPEG/PI fibers are obtained from CPEG/PAA fibers, so the paper only discusses the diameter variation of CPEG/PAA fibers.) The enhancement in the electrical conductivity increases the surface charge density of the CPEG/PAA jet and the electrostatic force received in the electric field, causing more jet splits and reducing the diameter of the prepared CPEG/ PAA fibers. However, the increase in surface tension makes it difficult to split the CPEG/PAA jet, so that the diameter of the CPEG/PAA fibers increases. When the amount of CPEG is $1 \mathrm{wt} \%$, the electrical conductivity of the CPEG/PAA solution increases greatly, reducing the diameter of the CPEG/PAA fibers. With the continuous increase of the amount of CPEG, the viscous stress of the CPEG/PAA solution is dominant. The effect of electrical conductivity weakens, and the diameter of the CPEG/PAA fibers increases. In addition, with the increase of CPEG amount, the number of beads in the CPEG/PI fibers gradually increases. The reason is that CPEG has high specific surface area, and the CNT on its surface has large length/diameter ratio, which makes CPEG easy to agglomerate. As the amount of CPEG increases, its volume fraction increases rapidly, which is not conducive to the uniform dispersion of CPEG in the CPEG/PAA solution, so that beads are formed in the CPEG/PAA fibers ${ }^{45}$. However, the overall dispersion of CPEG in the CPEG/PI fibers is relatively uniform (Fig. 3f), which is attributed to the shear effect of the CPEG/PAA solution passing through the needle during the electrospinning process and the high-speed stretching effect of the electric field on the CPEG/PAA jet. In addition, pure PI fibers and CPEG/PI fibers are oriented in the same direction, mainly ascribed to the self-made collection 
device of a cylinder with a copper wire frame. On the one hand, the cylinder rotates at high speed, and when its surface linear velocity is consistent with the settling velocity of fibers, a better orientation effect can be achieved. On the other hand, every two parallel wires on the cylinder can be regarded as parallel electrodes to induce fibers orientation ${ }^{46}$. The cross-section morphologies (Supplementary Fig. 5) of CPEG/PI composites have great changes with the amount of CPEG increases. The pure PI has a relatively flat fracture, and the partial domain is relatively rough, which means the PI is brittle and has a certain degree of toughness. With the addition of CPEG, the roughness and the number of folds in the CPEG/PI composites increase significantly, and there are lots of dimples fracture in the SEM images. It shows that the addition of CPEG helps to improve the toughness of $\mathrm{CPEG} / \mathrm{PI}$ composites. However, when the amount of CPEG reaches $10 \mathrm{wt} \%$, the number of dimples fracture is significantly reduced, and lamellar shape appears on the cross-section, indicating that the composites have a brittle fracture.

\section{Thermal conductivity of CPEG/PI composites}

Figure 4a shows the $\lambda$ of CEG/PI and CPEG/PI thermally conductive composites. It can be seen that the $\lambda$ of the two kinds of composites enhance with the increase in the amount of thermally conductive fillers (CEG or CPEG), and the $\lambda$ of the CPEG/PI thermally conductive composites has a faster growth rate. Under the same amount of thermally conductive fillers, the $\lambda$ of CPEG/PI thermally conductive composites is higher than that of CEG/PI thermally conductive composites. When the amount of CPEG is $10 \mathrm{wt} \%$, the $\lambda$ of CPEG/PI thermally conductive composites reaches $1.92 \mathrm{~W} \mathrm{~m}^{-1} \mathrm{~K}^{-1}$, which is about seven times that of pure $\mathrm{PI}$ $\left(0.28 \mathrm{~W} \mathrm{~m}^{-1} \mathrm{~K}^{-1}\right), 1.7$ times than that of $10 \mathrm{wt} \% \mathrm{CEG} / \mathrm{PI}$ thermally conductive composites $\left(1.12 \mathrm{~W} \mathrm{~m}^{-1} \mathrm{~K}^{-1}\right)$. The possible reasons are summarized as follows: (1) CPEG itself has high $\lambda$, and the 3D structure of the CNT pillared grown on EG gives it excellent inplane and through-plane $\lambda\left(\lambda_{\|}\right.$and $\left.\lambda_{\perp}\right)$, which is conducive to rapid
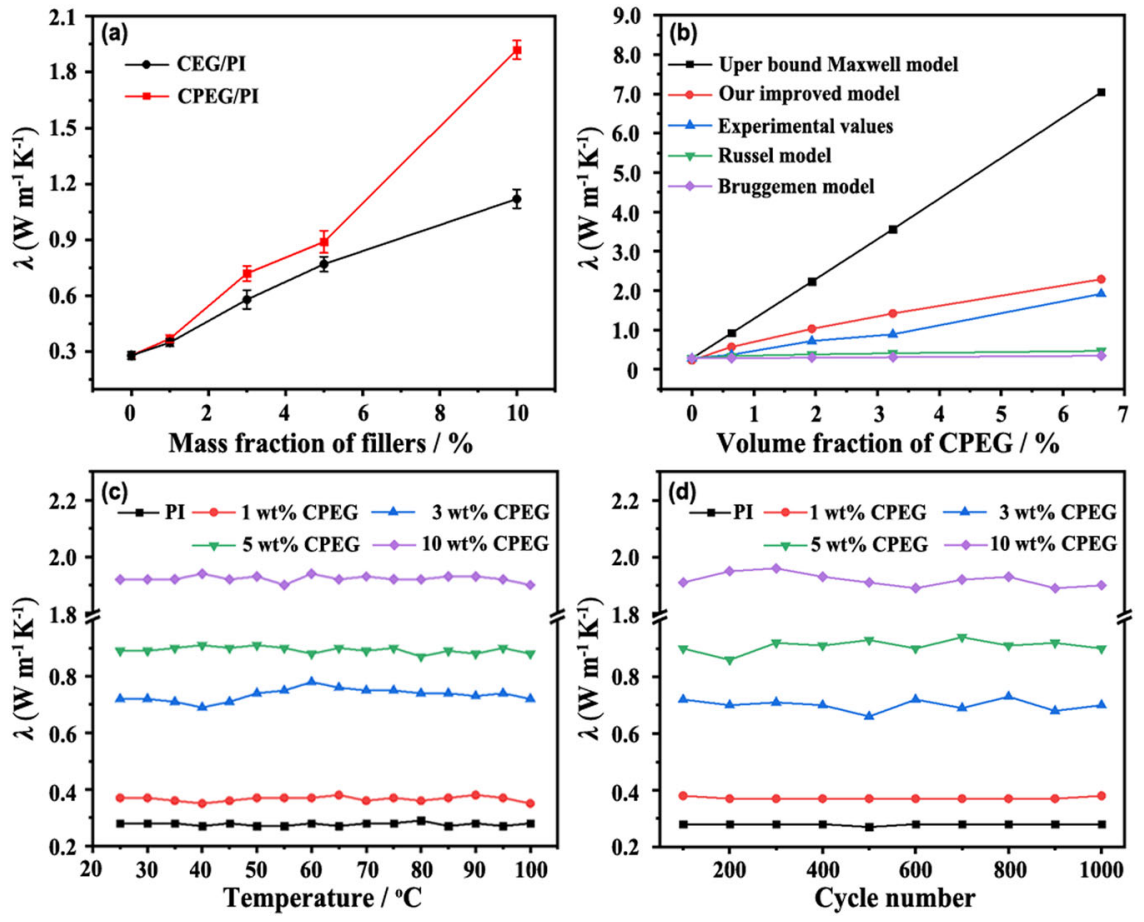

Fig. 4 Thermally conductive properties of CPEG/PI composites. The $\lambda$ of CEG/PI and CPEG/PI thermally conductive composites (a), the $\lambda$ calculated by the improved and classical thermal conductivity model and empirical equations (b), the influence of temperature on the $\lambda$ of CPEG/PI thermally conductive composites (c), the $\lambda$ of CPEG/PI thermally conductive composites experiencing heating and cooling cycles in the temperature range of $25-100^{\circ} \mathrm{C}\left(\mathbf{d}\right.$, test temperature of $\left.25^{\circ} \mathrm{C}\right)$.

heat transfer along the 3D direction ${ }^{47,48}$. (2) Electrospinning facilitates the orientation of $\mathrm{PI}$ molecular chains, reducing phonon scattering, and increasing the intrinsic $\lambda$ of the PI matrix. In addition, CPEG distributed on the PI fibers, the orientation and overlap of CPEG/PI fibers are beneficial to increasing the contact among CPEG, promoting the formation of CPEG-CPEG thermal conduction pathways, and increasing the $\lambda$ of CPEG/PI composites effectively ${ }^{49,50}$. The $\lambda$ of previous reports on CNT and graphite derivative filled polymer-based thermally conductive composites are summarized in Supplementary Table 1. It can be seen that the CPEG prepared in this paper has distinct strength in improving the $\lambda$ of polymer-based thermally conductive composites.

In order to deeply analyze the essential reasons that the CPEG/ PI thermally conductive composites have better thermal conductivity than CEG/PI thermally conductive composites, this paper utilizes COMSOL Multiphysics software to simulate the nano-/ microscale heat transfer in composites (Supplementary Note 3), the results are shown in Fig. 5. For CEG/PI composites, the temperature at $0.1 \mathrm{~s}$ is slightly higher than the temperature at $0.01 \mathrm{~s}$, and the temperature distribution is nonuniform. The CPEG/ PI thermally conductive composites have no temperature difference in 0.01 and $0.1 \mathrm{~s}$ and show the uniform distribution of temperature. In addition, at the same time point, the overall temperature of the CPEG/PI thermally conductive composites is higher than that of the CEG/PI thermally conductive composites. The main reason is that the CNT in the CEG is distributed randomly on the surface of the EG, and only some CNT interact with the EG through van der Waals forces, which results in high contact thermal resistance and does not conducive to thermal conduction (Fig. 5a). The overlap among CNT makes the heat transfer along the radial direction of CNT, which will cause thermal diffusion. Moreover, the low radial $\lambda$ of CNT and the high thermal resistance he contact point of CNT generate lots of heat loss, all of which result in non-uniform temperature distribution on the CNT (Fig. $5 \mathrm{~b}, \mathrm{c})$. In addition, when the heat is transferred from the CNT to $E G$, the thermal diffusion is extremely nonuniform due to the 
(a)

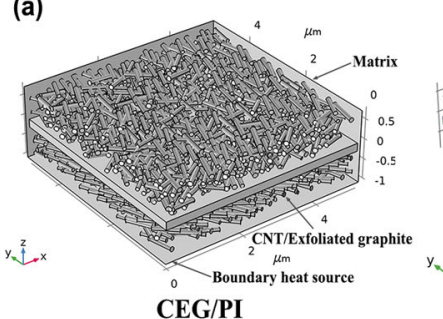

(d)

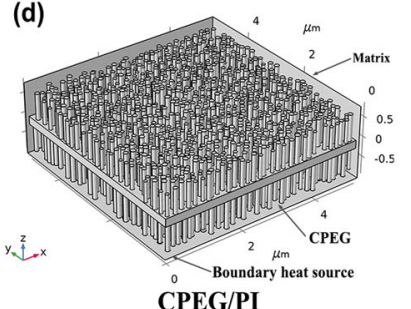

(b)

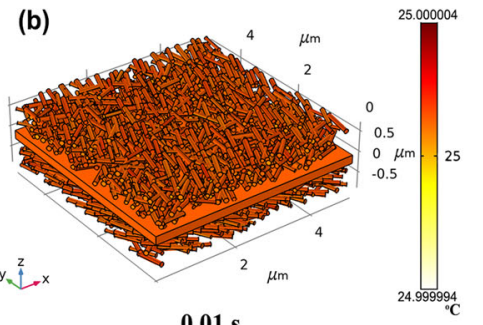

$0.01 \mathrm{~s}$

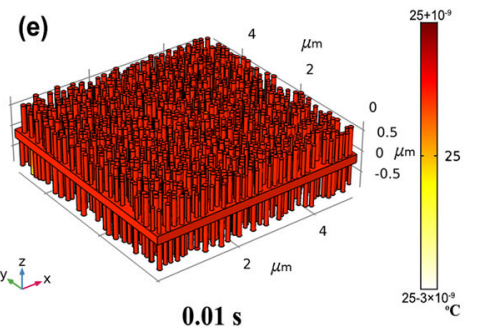

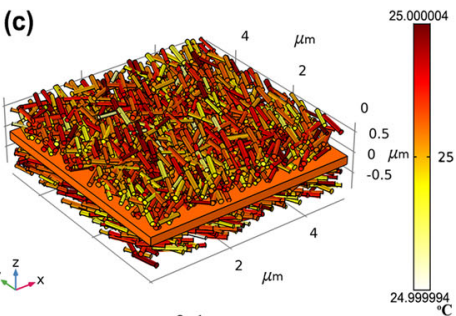

$0.1 \mathrm{~s}$

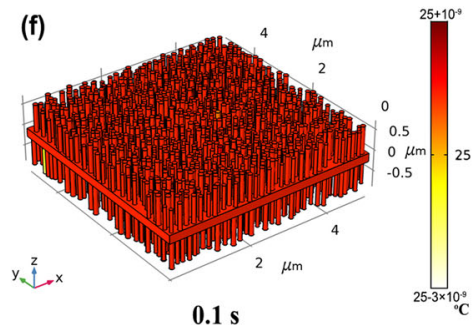

Fig. 5 Heat transfer simulation inside the PI-based composites. Inner structure of CEG/PI composites (a) and the temperature distribution at $0.01 \mathrm{~s} \mathrm{(b)} \mathrm{and} 0.1 \mathrm{~s}(\mathbf{c})$, inner structure of CPEG/PI composites (d), and the temperature distribution at $0.01 \mathrm{~s}(\mathbf{e})$ and $0.1 \mathrm{~s}(\mathbf{f})$.

partial contact between CNT and EG (Supplementary Fig. 6a), which makes a non-uniform temperature distribution in CEG (Fig. 5c). As for CPEG/PI thermally conductive composites, CNT and EG have high $\lambda$ in axial and in-plane directions, respectively. The 3D structure of CNT pillared grown on the EG sheet is beneficial to the thermal conduction to the surface of EG along the CNT axis (Fig. $5 \mathrm{~d})$, meanwhile, rapid heat diffusion in the EG sheet induces the temperature of CPEG to rise quickly. In addition, there is no interference among the oriented CNT, the CNT and EG are tightly bonded (Supplementary Fig. $6 \mathrm{~b}$ ) so that the heat loss in the CPEG is low. The high distribution density of CNT ensures that the heat can be conducted in time. All the advantages make sure uniform temperature distribution in CPEG (Fig. 5e, f).

In addition, in view of the narrow application range of existing thermal conductivity models and empirical equations in the calculation of the $\lambda$ of polymer-based thermally conductive composites. A more widely applicable thermal conductivity model and empirical equation for calculating the effective thermal conductivity of polymer-based composites is established based on the effective medium theory (EMT) ${ }^{51}$ and the law of conservation of energy (Supplementary Note 4).

$\lambda=\left[\frac{\lambda_{\|} \lambda_{\perp}\left(\lambda_{\|}^{2}+2 \lambda_{\|} \lambda_{\perp}\right)}{\pi \sqrt{\lambda_{\|}^{2}+2 \lambda_{\perp}^{2}}}\right]^{1 / 3}$

The theoretical $\lambda$ of CPEG/PI thermally conductive composites are calculated by Eq. 1 and classical empirical equations are shown in Fig. 4b. It can be seen that the thermal conductivity model and empirical equation established in this paper have a better fitting degree of theoretical calculation/experimental results than the classical empirical equations, and can better reflect the actual $\lambda$ change of CPEG/PI thermally conductive composites.

Figure $4 c$ shows the $\lambda$ of the CPEG/PI thermally conductive composites changes with testing temperature. It can be found that the variation trend of $\lambda$ with temperature is basically similar for CPEG/PI thermally conductive composites with different amount of CPEG. When the temperature increases from 25 to $100^{\circ} \mathrm{C}$, the $\lambda$ of the CPEG/PI thermally conductive composites is basically unchanged. In addition, the $\lambda$ stability of the composites after repeated heating and cooling cycles is very important to ensure the performance of the product. In this paper, the CPEG/PI thermally conductive composites are heated from 25 to $100^{\circ} \mathrm{C}$, and then cooled to $25^{\circ} \mathrm{C}$, and the $\lambda$ is tested at $25^{\circ} \mathrm{C}$ after every
100 cycles. The test results are shown in Fig. $4 \mathrm{~d}$. It can be clearly seen that the $\lambda$ of the CPEG/PI composites still maintains excellent stability after 1000 cycles in the temperature range of $25-100^{\circ} \mathrm{C}$, indicating its excellent environmental temperature tolerance. In addition, this paper tested the glass transition temperature $\left(T_{\mathrm{g}}\right)$ and heat resistance temperature $\left(T_{\mathrm{HRI}}\right)$ of CPEG/PI thermally conductive composites by DSC and TGA (Supplementary Fig. 7). The characteristic values are shown in Supplementary Table 2. It can be seen that the $5 \%$ weight loss temperature of CPEG/PI thermally conductive composites is higher than $500^{\circ} \mathrm{C}$, and its $T_{\mathrm{g}}$ and $T_{\mathrm{HRI}}$ both increase with the increase of the CPEG amount. The $T_{\mathrm{g}}$ and $T_{\mathrm{HRI}}$ of the CPEG/PI thermally conductive composites with $10 \mathrm{wt} \%$ CPEG are 214.7 and $293.0^{\circ} \mathrm{C}$, respectively, showing excellent heat resistance property. On the one hand, the $\mathrm{PI}$ backbone contains a large number of aromatic and imide rings, giving it high $T_{\mathrm{g}}$ and thermal decomposition temperature ${ }^{52,53}$. On the other hand, CPEG has high specific surface area, which restricts the movement of the $\mathrm{PI}$ molecular chains segments, increasing the $T_{\mathrm{g}}$ of the CPEG/PI composites ${ }^{54}$. Besides, the heat resistance of CPEG itself is higher than that of the $\mathrm{PI}$ matrix. The introduction of CPEG can improve the heat resistance of CPEG/PI composites. In addition, the formation of perfect 3D thermal conduction networks by CPEG will also facilitate the heat transfer and reduce the negative impact of the degradation of PI matrix due to local overheating caused by heat concentration.

In order to show the thermal conduction of the CPEG/PI thermally conductive composites more intuitively, this work places the CPEG/PI thermally conductive composites on a hot/cold plate with constant temperature and records the surface temperature by infrared thermography. The results are shown in Fig. 6a, b. It can be seen from Fig. $6 a$ that the color of the infrared images of the CPEG/PI thermally conductive composites gradually turns red with the heating time extension, indicating its surface temperature gradually increases. At the same time, the CPEG/PI thermally conductive composites with more CPEG amount have higher surface temperature, indicating that it has better $\lambda$. After the samples are heated up for $60 \mathrm{~s}$, the samples were quickly transferred to a $25^{\circ} \mathrm{C}$ stainless steel plate, and the surface temperature variation during the cooling process was recorded with the same infrared thermography. The relationship between the sample temperature and time is shown in Fig. $6 \mathrm{~b}$. It can be seen that the CPEG/PI thermally conductive composites with more CPEG amount not only have a faster heating rate, but also a higher 

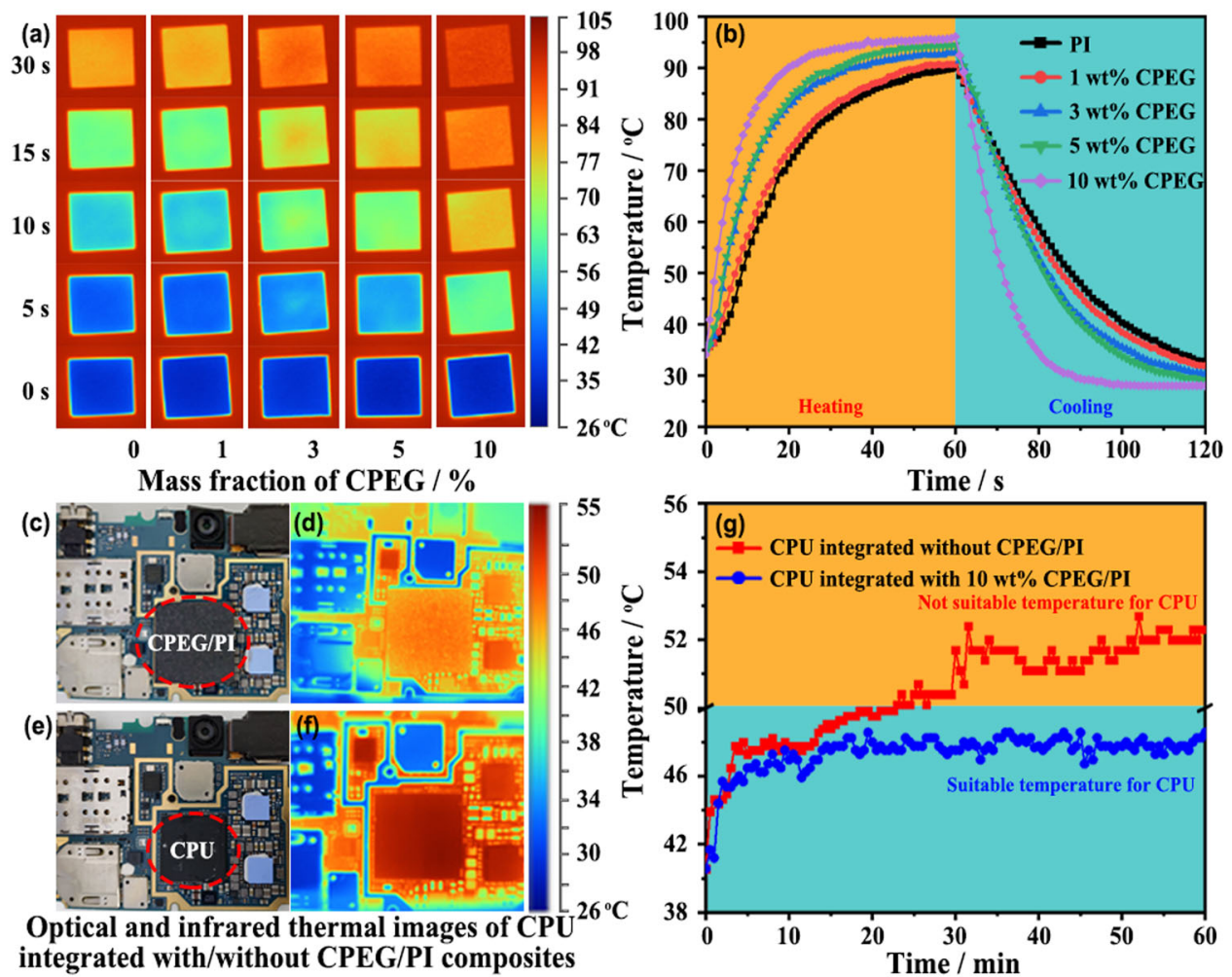

Fig. 6 Heat dissipation of CPEG/PI composites. Infrared images of CPEG/PI thermally conductive composites on the hot plate (a) and its surface temperature (b), optical photo (c) and infrared thermal images (d) of CPU integrated with CPEG/PI thermally conductive composites, optical photo (e) and infrared thermal images (f) of bare CPU, temperature of CPU during the working process (g).

surface temperature. Similarly, in the cooling process, the CPEG/PI thermally conductive composites containing more CPEG amount have a faster cooling rate and a lower surface temperature, indicating that the CPEG/PI thermally conductive composites prepared in this paper have excellent heat conduction and dissipation performance.

In order to further evaluate the heat conduction and dissipation performance of the CPEG/PI thermally conductive composites in practical applications. This work integrates the $10 \mathrm{wt} \% \mathrm{CPEG} / \mathrm{PI}$ thermally conductive composites with the CPU in mobile phone (Fig. 6c, d), and records the CPU temperature by CPU-Z software during the video playing period. After the phone is sufficiently cooled, removing the CPEG/PI thermally conductive composites to disclose the CPU (Fig. 6e, f) and playing the same video continuously for $1 \mathrm{~h}$. The relationship of the CPU temperature with time is shown in Fig. $6 \mathrm{~g}$. It can be seen that when the CPU temperature is stabilized, the temperature of the CPU integrated with CPEG/PI thermally conductive composites $\left(\sim 47.5^{\circ} \mathrm{C}\right)$ is significantly lower than that of the integrated without CPEG/PI thermally conductive composites $\left(\sim 52.3^{\circ} \mathrm{C}\right)$. It shows that the CPEG/PI thermally conductive composites have excellent heat conduction and dissipation capability as well as practical application value.

\section{METHODS}

\section{Preparation of CPEG}

Two grams of graphite, $80 \mathrm{~mL}$ of $\mathrm{H}_{2} \mathrm{SO}_{4}$, and $10 \mathrm{~g}$ of $\left(\mathrm{NH}_{4}\right)_{2} \mathrm{~S}_{2} \mathrm{O}_{8}$ were added into a flask, stirring evenly at $35^{\circ} \mathrm{C}$ until no bubble emission. Then, $200 \mathrm{~mL}$ of $\mathrm{H}_{2} \mathrm{O}$ was added into the flask drop by drop to ensure the temperature of the mixture under $60{ }^{\circ} \mathrm{C}$. The mixture was filtrated and washed several times until the filtrate was neutral, and then the filter cake was collected and dispersed in $100 \mathrm{~mL}$ of NMP and ultrasonicated (200 $\mathrm{KHz}$ ) for $1 \mathrm{~h}$. Finally, the exfoliated graphite (EG) was obtained after filtrating the NMP mixture followed by washing with deionized water and drying. After that, a certain amount of FC and EG were mixed uniformly and sealed in the quartz tube, put tube into the microwave oven $(900 \mathrm{~W}$, $2450 \mathrm{MHz}$ ), and CPEG with different morphologies were obtained by changing the gas atmosphere in the quartz tube and the microwave heating time.

\section{Fabrication of the CPEG/PI thermally conductive composites}

APB was dissolved in a mixed solvent of DMAc/THF (3/2, wt/wt), and a certain amount of CPEG was added and dispersed by ultrasonication. Under ice bath condition, a certain amount of ODPA (ODPA/APB $=1.01 / 1$, $\mathrm{mol} / \mathrm{mol}$ ) was added into the above solution and stirred for $4 \mathrm{~h}$ to prepare the CPEG/polyamide acid (CPEG/PAA) solution. The CPEG/PAA fibers mats were obtained after electrospinning the CPEG/PAA solution with the technology as follows: distance between the needle and collection device is $30 \mathrm{~cm}$, voltage of $20 \mathrm{kV}$, injection speed of $0.1 \mathrm{~mm} / \mathrm{min}$, and rotational speed of $700 \mathrm{rpm}$. The CPEG/PAA fibers mats were placed into the $80^{\circ} \mathrm{C}$ oven for $12 \mathrm{~h}$ for drying and then thermal imidization $\left(120^{\circ} \mathrm{C} / 1 \mathrm{~h}+200^{\circ} \mathrm{C} /\right.$ $1 \mathrm{~h}+250^{\circ} \mathrm{C} / 1 \mathrm{~h}$, heating rate of $1^{\circ} \mathrm{C} / \mathrm{min}$ ) to obtain CEPG/PI fibers mats. Finally, the CPEG/PI fibers mats were cut into $20^{*} 20 \mathrm{~mm}$ shape, then lay-up in a mold and hot-pressing at $320^{\circ} \mathrm{C} / 10 \mathrm{MPa}$ for $40 \mathrm{~min}$ to obtain the CPEG/PI thermally conductive composites $\left(20^{*} 20^{*} 2 \mathrm{~mm}\right)$. As a comparison, EG and purchased CNT were mixed directly in a certain ratio (CNT/EG $=3$ / 1 , wt/wt. This ratio is determined by the ratio of CNT to EG in CPEG) to obtain CNT/EG (CEG) thermally conductive fillers, and then CEG/PI thermally conductive composites were fabricated according to the above method.

\section{DATA AVAILABILITY}

The data that support the findings of this study are available from the corresponding author upon reasonable request. 
Received: 12 April 2021; Accepted: 28 June 2021; Published online: 15 July 2021

\section{REFERENCES}

1. Qian, X., Zhou, J. \& Chen, G. Phonon-engineered extreme thermal conductivity materials. Nat. Mater. https://doi.org/10.1038/s41563-021-00918-3 (2021).

2. Cui, Y., Qin, Z., Wu, H., Li, M. \& Hu, Y. Flexible thermal interface based on selfassembled boron arsenide for high-performance thermal management. Nat. Commun. 12, 1284 (2021).

3. Song, R. et al. Wideband and low sidelobe graphene antenna array for $5 \mathrm{G}$ applications. Sci. Bull. 66, 103-106 (2021).

4. Xiao, C. et al. Epoxy composite with significantly improved thermal conductivity by constructing a vertically aligned three-dimensional network of silicon carbide nanowires/ boron nitride nanosheets. Composites Part B 187, 107855 (2020).

5. Chen, J., Huang, X., Sun, B. \& Jiang, P. Highly thermally conductive yet electrically insulating polymer/boron nitride nanosheets nanocomposite films for improved thermal management capability. ACS Nano 13, 337-345 (2019).

6. Zhang, L., Deng, H. \& Fu, Q. Recent progress on thermal conductive and electrical insulating polymer composites. Compos. Commun. 8, 74-82 (2018).

7. Zhang, Y., Shen, Y., Shi, K., Wang, T. \& Harkin-Jones, E. Constructing a filler network for thermal conductivity enhancement in epoxy composites via reactioninduced phase separation. Composites Part A 110, 62-69 (2018).

8. Yang, X. et al. High-efficiency improvement of thermal conductivities for epoxy composites from synthesized liquid crystal epoxy followed by doping BN fillers. Composites Part B 185, 107784 (2020).

9. Yang, X., Zhong, X., Zhang, J. \& Gu, J. Intrinsic high thermal conductive liquid crystal epoxy film simultaneously combining with excellent intrinsic self-healing performance. J. Mater. Sci. Technol. 68, 209-215 (2021).

10. Ruan, K., Guo, Y. \& Gu, J. Liquid crystalline polyimide films with high intrinsic thermal conductivities and robust toughness. Macromolecules 54, 4934-4944 (2021).

11. Kang, D.-G. et al. Heat transfer organic materials: robust polymer films with the outstanding thermal conductivity fabricated by the photopolymerization of uniaxially oriented reactive discogens. ACS Appl. Mater. Interfaces 8, 30492-30501 (2016).

12. $\mathrm{Xu}, \mathrm{Y}$. et al. Molecular engineered conjugated polymer with high thermal conductivity. Sci. Adv. 4, eaar3031 (2018).

13. $\mathrm{Li}, \mathrm{C}$. et al. Three-dimensional interconnected graphene microsphere as fillers for enhancing thermal conductivity of polymer. Chem. Eng. J. 368, 79-87 (2019).

14. Wang, Y.-Y. et al. Lightweight and robust carbon nanotube/polyimide foam for efficient and heat-resistant electromagnetic interference shielding and microwave absorption. ACS Appl. Mater. Interfaces 12, 8704-8712 (2020).

15. Tan, C. et al. A high performance wearable strain sensor with advanced thermal management for motion monitoring. Nat. Commun. 11, 3530 (2020).

16. Zhou, H., Zhang, Z., Sun, C., Deng, H. \& Fu, Q. Biomimetic approach to facilitate the high filler content in free-standing and flexible thermoelectric polymer composite films based on PVDF and $\mathrm{Ag}_{2}$ Se nanowires. ACS Appl. Mater. Interfaces 12, 51506-51516 (2020).

17. Song, Y., Jiang, F., Song, N., Shi, L. \& Ding, P. Multilayered structural design of flexible films for smart thermal management. Composites Part A 141, 106222 (2021).

18. Zhang, F. et al. Stress controllability in thermal and electrical conductivity of $3 D$ elastic graphene-crosslinked carbon nanotube sponge/polyimide nanocomposite. Adv. Funct. Mater. 29, 1901383 (2019).

19. Guo, Y., Ruan, K., Shi, X., Yang, X. \& Gu, J. Factors affecting thermal conductivities of the polymers and polymer composites: a review. Compos. Sci. Technol. 193, 108134 (2020).

20. Jiang, F., Cui, X., Song, N., Shi, L. \& Ding, P. Synergistic effect of functionalized graphene/boron nitride on the thermal conductivity of polystyrene composites. Compos. Commun. 20, 100350 (2020).

21. $\mathrm{Hu}, \mathrm{D}$. M. et al. Strong graphene-interlayered carbon nanotube films with high thermal conductivity. Carbon 118, 659-665 (2017).

22. Song, P. et al. Lightweight, flexible cellulose-derived carbon aerogel@reduced graphene oxide/PDMS composites with outstanding EMI shielding performances and excellent thermal conductivities. Nano-Micro Lett. 13, 91 (2021).

23. Xiao, Y. J. et al. Hybrid network structure and thermal conductive properties in poly(vinylidene fluoride) composites based on carbon nanotubes and graphene nanoplatelets. Composites Part A 90, 614-625 (2016).

24. Yu, J., Choi, H. K., Kim, H. S. \& Kim, S. Y. Synergistic effect of hybrid graphene nanoplatelet and multi-walled carbon nanotube fillers on the thermal conductivity of polymer composites and theoretical modeling of the synergistic effect. Composites Part A 88, 79-85 (2016).
25. Guo, H. et al. High thermal conductive poly(vinylidene fluoride)-based composites with well-dispersed carbon nanotubes/graphene three-dimensional network structure via reduced interfacial thermal resistance. Compos. Sci. Technol. 181, 107713 (2019).

26. He, L. Improve thermal conductivity of polymer composites via conductive network. ES Mater. Manuf. 13, 1-2 (2021).

27. Jha, S. K. et al. First-principles study of the interactions between graphene oxide and amine-functionalized carbon nanotube. J. Phys. Chem. C. 122, 1288-1298 (2018).

28. Wang, Z.-G. et al. Achieving excellent thermally conductive and electromagnetic shielding performance by nondestructive functionalization and oriented arrangement of carbon nanotubes in composite films. Compos. Sci. Technol. 194, 108190 (2020).

29. Cao, M. et al. Paving thermally conductive highway by 3D interconnected framework of carbon nanotube and graphene oxide in poly(vinylidene fluoride). Composites Part A 115, 331-340 (2018).

30. Zhao, C. B. et al. Thermally conductive cyanate ester nanocomposites filled with graphene nanosheets and multiwalled carbon nanotubes. Polym. Adv. Technol. 25, 1546-1551 (2014).

31. Guo, Y. et al. Constructing fully carbon-based fillers with a hierarchical structure to fabricate highly thermally conductive polyimide nanocomposites. J. Mater. Chem. C. 7, 7035-7044 (2019).

32. Ruan, K., Shi, X., Guo, Y. \& Gu, J. Interfacial thermal resistance in thermally conductive polymer composites: a review. Compos. Commun. 22, 100518 (2020).

33. Zhang, Z., Qu, J., Feng, Y. \& Feng, W. Assembly of graphene-aligned polymer composites for thermal conductive applications. Compos. Commun. 9, 33-41 (2018).

34. Sun, S. et al. Improving thermal transport at carbon hybrid interfaces by covalent bonds. Adv. Mater. Interfaces 5, 1800318 (2018).

35. Qin, M. M., Feng, Y. Y., Ji, T. X. \& Feng, W. Enhancement of cross-plane thermal conductivity and mechanical strength via vertical aligned carbon nanotube@graphite architecture. Carbon 104, 157-168 (2016).

36. Zhan, H., Nie, Y., Chen, Y., Bell, J. M. \& Gu, Y. Thermal transport in 3D nanostructures. Adv. Funct. Mater. 30, 1903841 (2019).

37. Lv, F. et al. High cross-plane thermally conductive hierarchical composite using graphene-coated vertically aligned carbon nanotubes/graphite. Carbon 149, 281-289 (2019).

38. Kumar, R. et al. Self-assembled nanostructures of 3D hierarchical faceted-iron oxide containing vertical carbon nanotubes on reduced graphene oxide hybrids for enhanced electromagnetic interface shielding. Composites Part B 168, 66-76 (2019).

39. Hazarika, A., Deka, B. K., Kim, D., Park, Y. B. \& Park, H. W. Microwave-induced hierarchical iron-carbon nanotubes nanostructures anchored on polypyrrole/ graphene oxide-grafted woven Kevlar (R) fiber. Compos. Sci. Technol. 129, 137-145 (2016).

40. Zhang, $H$. et al. Metallic sandwiched-aerogel hybrids enabling flexible and stretchable intelligent sensor. Nano Lett. 20, 3449-3458 (2020).

41. Kwon, Y. J., Kwon, Y., Park, H. S. \& Lee, J. U. Mass-produced electrochemically exfoliated graphene for ultrahigh thermally conductive paper using a multimetal electrode system. Adv. Mater. Interfaces 6, 1900095 (2019).

42. Zhang, R., Zhang, Y. \& Wei, F. Controlled synthesis of ultralong carbon nanotubes with perfect structures and extraordinary properties. Acc. Chem. Res. 50, 179-189 (2017).

43. Su, Q., Li, J., Du, G. \& Xu, B. In situ TEM study on the electrical and field-emission properties of individual $\mathrm{Fe}_{3} \mathrm{C}$-filled carbon nanotubes. J. Phys. Chem. C. 116, 23175-23179 (2012).

44. Lozzi, L., Passacantando, M., Picozzi, P., Santucci, S. \& Dendaas, H. Oxidation of the FE/CU(100) interface. Surf. Sci. 331, 703-709 (1995).

45. Bao, Q. et al. Graphene-polymer nanofiber membrane for ultrafast photonics. Adv. Funct. Mater. 20, 782-791 (2010).

46. Ruan, K., Zhong, X., Shi, X., Dang, J. \& Gu, J. Liquid crystal epoxy resins with high intrinsic thermal conductivities and their composites: A mini-review. Mater. Today Phys. 20, 100456 (2021).

47. Shi, X. et al. Improvement of thermal conductivities and simulation model for glass fabrics reinforced epoxy laminated composites via introducing heterostructured BNN-30@BNNS fillers. J. Mater. Sci. Technol. 82, 239-249 (2021).

48. Zhou, Y., Liu, F. \& Chen, C.-Y. Use of BN-coated copper nanowires in nanocomposites with enhanced thermal conductivity and electrical insulation. Adv. Compos. Hybrid. Mater. 2, 46-50 (2019).

49. Guo, Y., Ruan, K. \& Gu, J. Controllable thermal conductivity in composites by constructing thermal conduction networks. Mater. Today Phys. 20, 100449 (2021).

50. Gu, J. \& Ruan, K. Breaking through bottlenecks for thermally conductive polymer composites: a perspective for intrinsic thermal conductivity, interfacial thermal resistance and theoretics. Nano-Micro Lett. 13, 110 (2021). 
51. Nan, C. W., Birringer, R., Clarke, D. R. \& Gleiter, H. Effective thermal conductivity of particulate composites with interfacial thermal resistance. J. Appl. Phys. 81, 6692-6699 (1997).

52. Yang, F. et al. Superhydrophobic polyvinylidene fluoride/polyimide nanofiber composite aerogels for thermal insulation under extremely humid and hot environment. Sci. China Mater. 64, 1-11 (2020).

53. Gao, C., Shen, Y. \& Wang, T. Enhanced thermal conductivity for traditional epoxy packaging composites by constructing hybrid conductive network. Mater. Res. Express 7, 065308 (2020).

54. Ma, J. et al. Effect of inorganic nanoparticles on mechanical property, fracture toughness and toughening mechanism of two epoxy systems. Polymer 49, 3510-3523 (2008).

\section{ACKNOWLEDGEMENTS}

This work was supported by the National Natural Science Foundation of China (51973173 and 51773169); Guangdong Basic and Applied Basic Research Foundation (2019B1515120093); Technical Basis Scientific Research Project (Highly Thermally Conductive Nonmetal Materials); Natural Science Basic Research Plan for Distinguished Young Scholars in Shaanxi Province of China (2019JC-11); the Innovation Foundation for Doctor Dissertation of Northwestern Polytechnical University (CX202055); Polymer Electromagnetic Functional Materials Innovation Team of Shaanxi Sanqin Scholars; the Analytical and Testing Center of Northwestern Polytechnical University.

\section{AUTHOR CONTRIBUTIONS}

All authors contributed to the preparation of this paper. J.G. and Y.G. conceived and designed the experiments. Y.G., S.W., and K.R. characterized and analyzed the materials. Y.G., S.W., K.R., and H.Z. contributed to the writing of the paper. J.G. supervised the overall research.

\section{COMPETING INTERESTS}

The authors declare no competing interests.

\section{ADDITIONAL INFORMATION}

Supplementary information The online version contains supplementary materia available at https://doi.org/10.1038/s41528-021-00113-z.

Correspondence and requests for materials should be addressed to J.G.

Reprints and permission information is available at http://www.nature.com/ reprints

Publisher's note Springer Nature remains neutral with regard to jurisdictional claims in published maps and institutional affiliations.

Open Access This article is licensed under a Creative Commons Attribution 4.0 International License, which permits use, sharing, adaptation, distribution and reproduction in any medium or format, as long as you give appropriate credit to the original author(s) and the source, provide a link to the Creative Commons license, and indicate if changes were made. The images or other third party material in this article are included in the article's Creative Commons license, unless indicated otherwise in a credit line to the material. If material is not included in the article's Creative Commons license and your intended use is not permitted by statutory regulation or exceeds the permitted use, you will need to obtain permission directly from the copyright holder. To view a copy of this license, visit http://creativecommons. org/licenses/by/4.0/.

C The Author(s) 2021 